\title{
An Efficient Gait Recognition with Backpack Removal
}

\author{
Heesung Lee, Sungjun Hong, and Euntai Kim \\ Biometrics Engineering Research Center, School of Electrical and Electronic Engineering, Yonsei University, \\ Sinchon-dong, Seodaemun-gu, Seoul 120-749, South Korea
}

Correspondence should be addressed to Euntai Kim, etkim@yonsei.ac.kr

Received 12 February 2009; Accepted 12 August 2009

Recommended by Moon Kang

Gait-based human identification is a paradigm to recognize individuals using visual cues that characterize their walking motion. An important requirement for successful gait recognition is robustness to variations including different lighting conditions, poses, and walking speed. Deformation of the gait silhouette caused by objects carried by subjects also has a significant effect on the performance of gait recognition systems; a backpack is the most common of these objects. This paper proposes methods for eliminating the effect of a carried backpack for efficient gait recognition. We apply simple, recursive principal component analysis (PCA) reconstructions and error compensation to remove the backpack from the gait representation and then conduct gait recognition. Experiments performed with the CASIA database illustrate the performance of the proposed algorithm.

Copyright ( $) 2009$ Heesung Lee et al. This is an open access article distributed under the Creative Commons Attribution License, which permits unrestricted use, distribution, and reproduction in any medium, provided the original work is properly cited.

\section{Introduction}

Gait recognition is the identification of individuals based on their walking style [1]. The theoretic foundation of gait recognition is the uniqueness of each person's gait, as revealed by Murray et al. in 1964 [2]. Gait analysis has the advantage of being noninvasive and noncontact. Gait is also less likely to be obscured than other biometrics such as face, fingerprints, and iris. Furthermore, gait is the only biometric which can be perceived at a long distance [3]. Hence, the gait recognition system has recently attracted increasing interest from researchers in the field of computer vision. Gait recognition methods can be classified into two broad types: model-based and silhouette-based approaches [4].

Model-based approaches try to represent the human body or motion precisely by employing explicit models describing gait dynamics, such as stride dimensions and the kinematics of joint angles [5-7]. The effectiveness of model-based approaches, however, is still limited due to imperfect vision techniques in body structure/motion modeling and parameter recovery from a walking image sequence. Moreover, precise modeling makes model-based approaches computationally expensive.
By contrast, the silhouette-based approaches characterize body movement using statistics of the walking patterns which capture both static and dynamic properties of body shape [8-15]. In these approaches, the representation methods for human gait obviously play a critical part. Several methods of this type have been reported, for example, gait energy image (GEI) [8], motion silhouette image (MSI) [9], motion history image (MHI) [10], tensor data [11-13], mass profile [14], and so forth. GEI is the most popular silhouette-based gait representation method and exhibits good performance and robustness against segmental error [8]. As a variation, Tan et al. used the head-torso-thigh part of human silhouettes to represent human gait [15]. This is actually a part of GEI and is called HTI. HTI is more robust against variation in walking speed than GEI. In gait recognition, one important requirement is robustness to variations including lighting conditions, poses, and walking speed. The deformation of the gait silhouette caused by carried objects also has a significant effect on the performance of gait recognition systems; a backpack is the most common of these objects.

In this paper, we propose a backpack removal method for efficient and robust gait recognition. We employ the silhouette-based approach. Even though HTI is more robust 


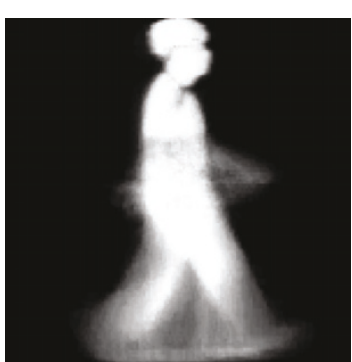

(a)

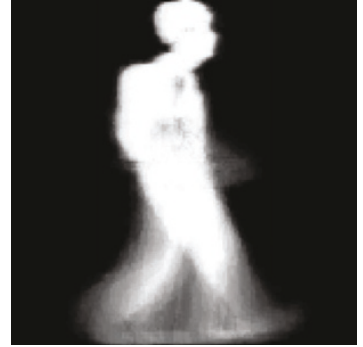

(b)

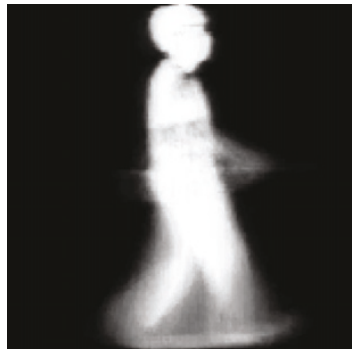

(c)

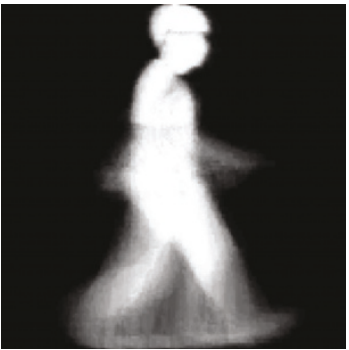

(d)

FIGURE 1: GEI for (a) walking normally, (b) walking with a backpack, (c) walking slowly, and (d) walking quickly.

than GEI with respect to the walking speed, it performs poorly when a backpack is involved. For this reason, we use GEI as a gait representation method and apply simple, recursive principal component analysis (PCA) reconstructions $[16,17]$ and error compensation to remove a backpack from GEI. We build the principal components from the training GEIs without a backpack and recover a new GEI with a backpack using the backpack-free principal components. Because the representational power of PCA depends on the training set, the PCA removes the backpack using the backpack-free principal components. Two studies were reported regarding gait recognition while the subject held or carried an object. In [18], GEI was decomposed into supervised and unsupervised parts and applied to gait recognition while the individual carried a coat and a small bag. In [19], the robust gait feature based on the general tensor discriminant analysis (GTDA) was proposed to cope with the silhouette deformation caused by a briefcase. Our work has a similar goal to that of $[18,19]$. However, our study does not compete with these two other studies, but rather complements them, because our method may be used as a preprocessing step before either [18] or [19] is applied.

This paper is organized as follows. In Section 2, we provide some background, about gait representations and the database used for the experiments. In Section 3, backpack removal methods based on simple and recursive PCA reconstructions are presented. In Section 4, the proposed methods are applied to the Chinese Academy of Sciences (CASIA) gait dataset $\mathrm{C}$, and its performance is compared with those of other methods. Conclusions are drawn in Section 5.

\section{Background}

2.1. Gait Energy Image. Gait representations are of crucial importance in the performance of gait recognition. GEI is an effective representation scheme with good discriminating power and robustness against segmental errors [8]. Given the preprocessed binary gait silhouette images $B_{t}(x, y)$ at time $t$ in a sequence, GEI is computed by

$$
G(x, y)=\frac{1}{N} \sum_{t=1}^{N} B_{t}(x, y)
$$

where $N$ is the number of frames in the complete gait sequence, and $x$ and $y$ are values in the image coordinates. Figure 1 shows some examples GEI. In comparison with gait representation using a binary silhouette sequence, GEI saves both storage space and computation time for recognition and is less sensitive to noise in individual silhouette images. When a silhouette is deformed, however, even GEI exhibits degraded performance. Backpacks are one of the most common objects that significantly deform a silhouette. Therefore, we propose new methods which remove the backpacks in GEIs.

2.2. Database. In this paper we use the CASIA dataset $C$ [15]. In the database, each subject has ten walking sequences: he/she walks normally four times, walks slowly twice, and walks quickly twice, all without a backpack, and then walks at a normal speed with a backpack twice. The database has 153 subjects (130 males and 23 females) and thus includes a total of $153 \times 10=1530$ walking sequences. This database was initially invented for infrared-based gait recognition and night visual surveillance. But this is irrelevant for our research, since we use only binarized gait silhouettes. Figures 2 and 3 show some original and normalized example images of this database, respectively. The original silhouette images are normalized to $R^{q}$ based on the height of the subject, where $q$ denotes the size of normalized silhouette images and it is fixed to $120 \times 120$.

\section{Backpack Removal Using PCA Reconstruction}

In this section, we propose two backpack removal methods. The first is based on a simple PCA and the other on a recursive PCA. When a GEI with a backpack is given, we aim to generate a new GEI without the backpack while keeping the rest of the image intact. The basic idea of backpack removal is to reconstruct the GEI with a backpack using the principal components (eigenvectors) of the GEIs without a backpack. Since the principal components are computed from GEIs without a backpack, they should have no capacity (information) to represent or recover the backpack region in the GEI. Thus, when a GEI is given and reconstructed using the backpack-free principal components, the resulting 

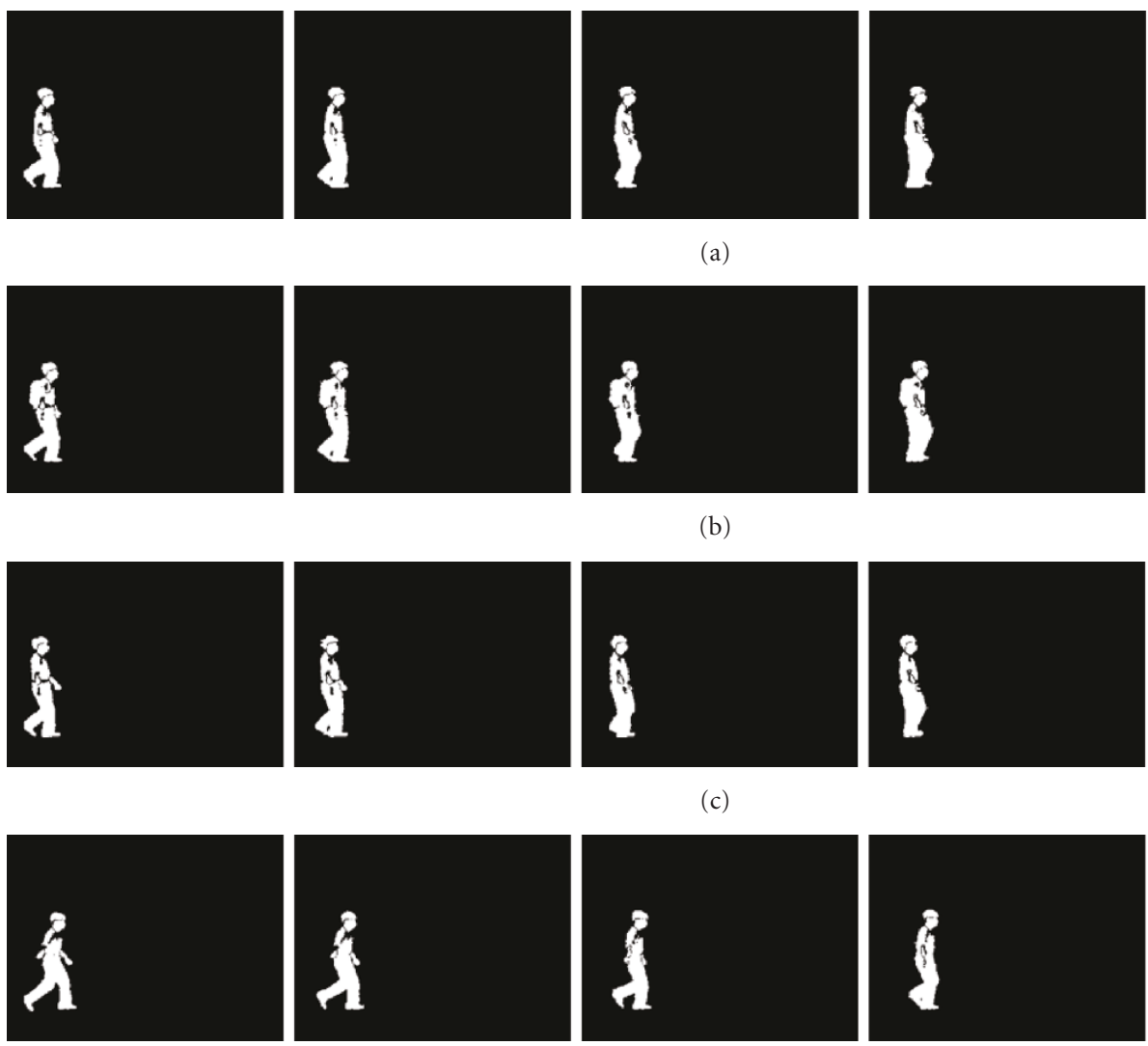

(d)

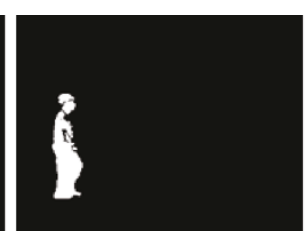

(a)
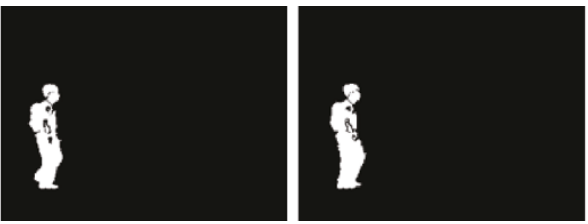

(b)
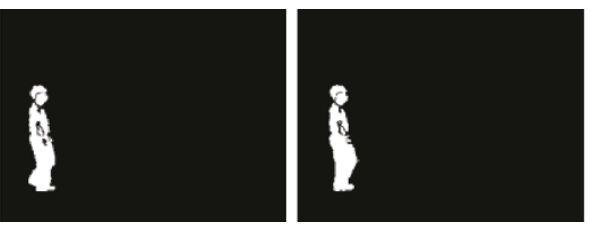

(c)
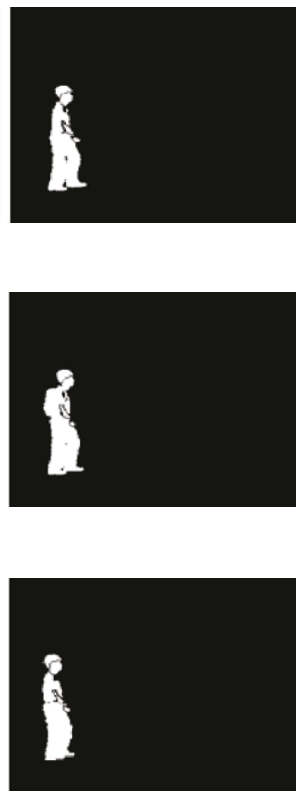

FIgURE 2: Examples of original images: (a) walking normally, (b) walking with a backpack, (c) walking slowly, and (d) walking quickly.

image should be a new GEI without a backpack. This idea is motivated by $[16,17]$.

3.1. Backpack Removal Using Simple PCA Reconstruction. We denote the training GEIs without a backpack by $G_{w / o}(i) \in$ $R^{q},(i=1, \ldots, l)$, where $l$ is the number of training images and $q$ is the number of pixels of each GEI. The average and the covariance of the images are defined as

$$
\begin{gathered}
\mu=\frac{1}{l} \sum_{i=1}^{l} G_{w / o}(i), \\
\Sigma=\frac{1}{l} \sum_{i=1}^{l}\left(G_{w / o}(i)-\mu\right)\left(G_{w / o}(i)-\mu\right)^{T},
\end{gathered}
$$

respectively. A projection matrix $P_{w / o}$ is chosen to maximize the determinant of the covariance matrix of the projected images, that is,

$$
P_{w / o}=\left[P_{w / o}^{1} P_{w / o}^{2} \cdots P_{w / o}^{q}\right]=\arg \max _{P}\left|P^{T} \Sigma P\right|,
$$

where $\left\{P_{w / o}^{t} \mid t=1,2, \ldots, q\right\}$ is the set of $q$-dimensional eigenvectors of the covariance matrix. When a new input GEI
$G$ with a backpack is given, it is projected using $P_{w / o}$ and reconstructed by

$$
\begin{aligned}
G^{R} & =\mu+P_{w / o}\left(P_{w / o}^{T}(G-\mu)\right) \\
& =\mu+\left[P_{w / o}^{1} P_{w / o}^{2} \cdots P_{w / o}^{q}\right]\left(\left[P_{w / o}^{1} P_{w / o}^{2} \cdots P_{w / o}^{q}\right]^{T}(G-\mu)\right),
\end{aligned}
$$

where $G^{R}$ is the reconstructed GEI of $G$. Since the projection matrix $P_{w / o}$ is derived from the GEIs without a backpack and has no information about the backpack region in the GEI, the $G^{R}$ recovered from $G$ has no backpack. In the reconstruction process, it is likely that some errors caused by backpack removal are spread out over the entire image and degrade the quality of image. We thus combine the left half of the backpack-removed image with the right half of the original image by

$$
G^{C}(x, y)= \begin{cases}G^{R}(x, y), & \text { if }(x, y) \in \text { left part of the image, } \\ G(x, y), & \text { otherwise, }\end{cases}
$$

where $G^{C}$ is the error-compensated, reconstructed image. The results are shown in Figure 4. In Figure 4, it can be 

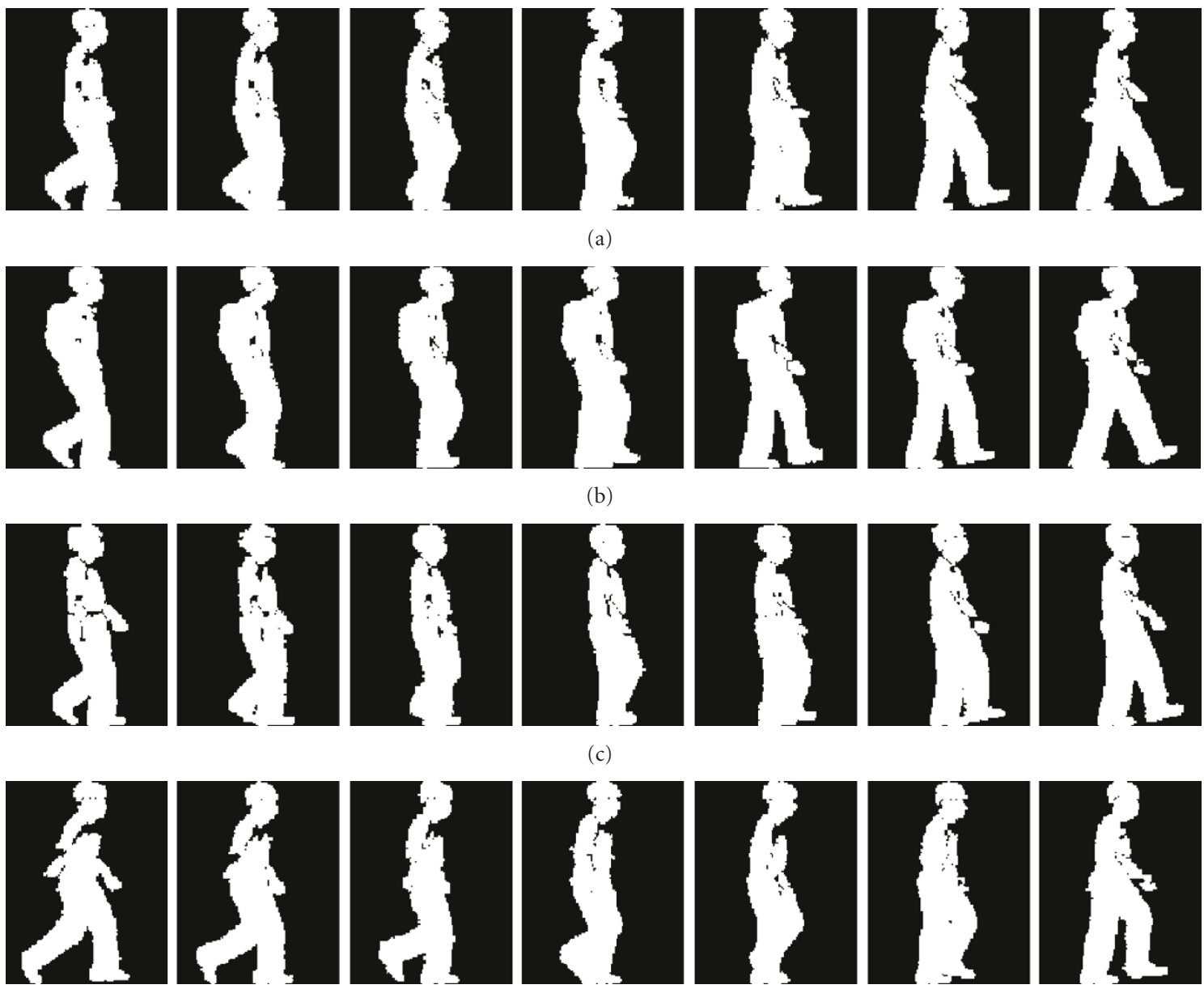

(d)

FIGURE 3: Examples of normalized images: (a) walking normally, (b) walking with a backpack, (c) walking slowly, and (d) walking quickly.

observed that the quality of the reconstructed image is improved, especially around the head region of the GEI.

\subsection{Backpack Removal Using Recursive PCA Reconstruction.}

If a large area of GEI is affected by a backpack and the backpack is removed by a simple PCA, the resulting reconstructed GEI often retains some traces of the backpack. In this section, we apply the recursive PCA reconstruction to remove a backpack from the gait image. By iterating the projection onto the backpack-free components $P_{w / o}$, we process the backpack region recursively, obtaining a GEI in which the backpack is more clearly removed. This approach is motivated by [17]. As stated above, the original GEI $G$ with a backpack is projected using $P_{w / o}$ and reconstructed into $G_{1}^{R}$ by

$$
\begin{aligned}
G_{1}^{R} & =\mu+P_{w / o}\left(P_{w / o}^{T}(G-\mu)\right) \\
& =\mu+\left[P_{w / o}^{1} P_{w / o}^{2} \cdots P_{w / o}^{q}\right]\left(\left[P_{w / o}^{1} P_{w / o}^{2} \cdots P_{w / o}^{q}\right]^{T}(G-\mu)\right) .
\end{aligned}
$$

Then, the difference between $G$ and its reconstructed version $G_{1}^{R}$ is computed by

$$
d_{1}(x, y)=\left|G(x, y)-G_{1}^{R}(x, y)\right|
$$

Since $G_{1}^{R}$ is reconstructed using backpack-free components $P_{w / o}$ and the backpack is almost removed, $d_{1}(x, y)$ becomes large around the backpack region of the reconstructed GEI. Using $d_{1}(x, y)$, we locate the backpack region in $G_{1}^{R}$ and compensate the other region by

$$
\begin{gathered}
G_{1}^{R C}(x, y)=\lambda_{1}(x, y) G_{1}^{R}(x, y)+\left(1-\lambda_{1}(x, y)\right) G(x, y), \\
\lambda_{1}(x, y)=1, \quad d_{1}(x, y) \geq \xi_{h}, \\
\lambda_{1}(x, y)=\frac{d_{1}(x, y)-\xi_{l}}{\xi_{h}-\xi_{l}}, \quad \xi_{l} \leq d_{1}(x, y)<\xi_{h}, \\
\lambda_{1}(x, y)=0, \quad d_{1}(x, y)<\xi_{l},
\end{gathered}
$$

where $G_{1}^{R C}$ is a new reconstructed image, $\lambda_{1}$ is the weight for error compensation, and $\xi_{h}$ and $\xi_{l}$ are the thresholds for 

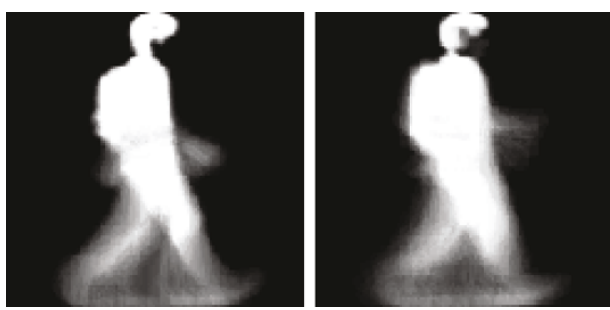

(a)
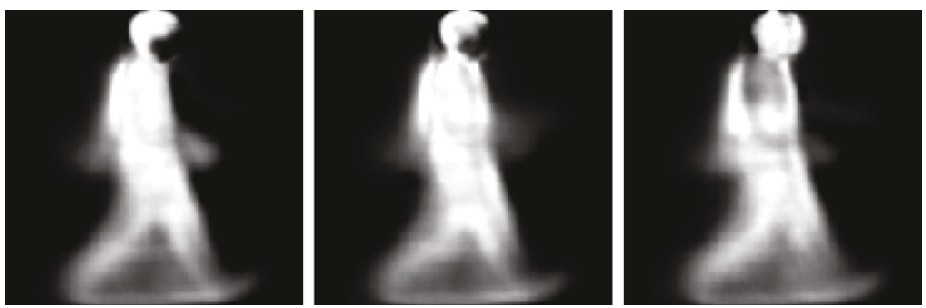

(b)
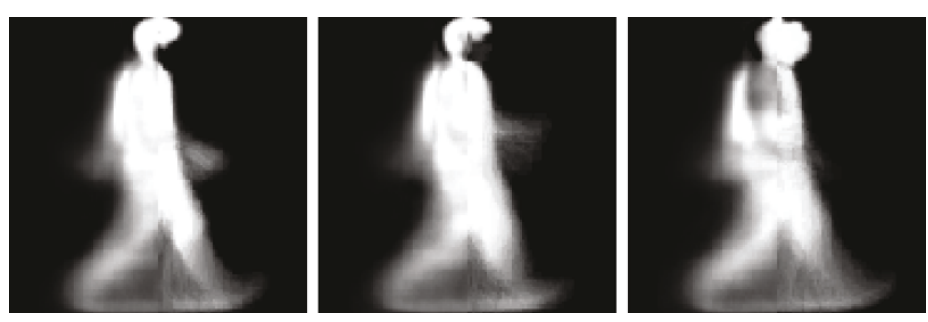

(c)

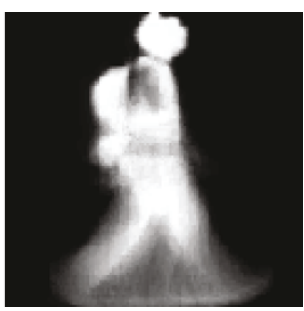

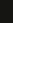

FIGURE 4: Result of backpack removal using simple PCA reconstruction: (a) original images with backpack, (b) reconstructed images without a backpack removed by PCA, and (c) images built by combining right part of (a) and left part of (b).

the backpack region and non-backpack region, respectively. We repeat the same error compensation procedure:

$$
\begin{aligned}
& G_{t}^{R}=\mu+P_{w / o}\left(P_{w / o}^{T}\left(G_{t-1}^{R C}-\mu\right)\right) \\
& =\mu+\left[P_{w / 0}^{1} P_{w / o}^{2} \cdots P_{w / o}^{q}\right] \\
& \times\left(\left[P_{w / o}^{1} P_{w / o}^{2} \cdots P_{w / o}^{q}\right]^{T}\left(G_{t-1}^{R C}-\mu\right)\right), \\
& d_{t}(x, y)=\left|G(x, y)-G_{t}^{R}(x, y)\right|, \\
& \lambda_{t}(x, y)= \begin{cases}1, & \text { if } d_{t}(x, y) \geq \xi_{h}, \\
\frac{d_{t}(x, y)-\xi_{l}}{\xi_{h}-\xi_{l}}, & \text { if } \xi_{l} \leq d_{t}(x, y)<\xi_{h}, \\
0, & \text { if } d_{t}(x, y)<\xi_{l},\end{cases} \\
& G_{t}^{R C}(x, y)=\lambda_{t}(x, y) G_{t}^{R}(x, y) \\
& +\left(1-\lambda_{t}(x, y)\right) G(x, y) \text { for } t>1,
\end{aligned}
$$

until the difference between the currently compensated GEI $\left(G_{t}^{R C}\right)$ and the previously compensated GEI $\left(G_{t-1}^{R C}\right)$ falls below a threshold:

$$
\left\|G_{t}^{R C}-G_{t-1}^{R C}\right\| \leq \varepsilon
$$

Here, $t$ is the iteration index, $G_{t}^{R C}(x, y)$ is a compensated GEI at the th iteration, and $G_{t}^{R}(x, y)$ is a temporary image at the tth iteration reconstructed from $G_{t-1}^{R C}(x, y)$. The results of backpack removal using recursive PCA reconstruction are shown in Figure 5.

\section{Experiments}

In this section, we apply the suggested backpack removal methods to the CASIA database to show their effectiveness. To compute the projection matrix $P_{w / o}$ and the average image $\mu$ of the gaits without a backpack, we use the eight sequences of normal, slow, and quick walking GEIs as the training set of PCA. The differences $d_{1}(x, y)=\left|G(x, y)-G_{1}^{R}(x, y)\right|$ between the original image $G(x, y)$ and the associated reconstruction image $G_{1}^{R}(x, y)$ are collected from several sample images, and the differences are divided into two groups depending on whether the associated pixel belongs to the backpack region or the nonbackpack region. For the pixels in the backpack region, the differences $d_{1}(x, y)$ are close to 1 and $\xi_{h}$ is selected such that $90 \%$ of the pixels satisfy $d_{1}(x, y) \geq \xi_{h}$. Similarly, the differences $d_{1}(x, y)$ are close to 0 for the pixels in the nonbackpack region and $\xi_{l}$ is selected such that $90 \%$ of the pixels satisfy $d_{1}(x, y)<\xi_{l}$. We employ the 1-Nearest Neighborhood (1-NN) as a classifier and use the sequences of normal walking as the training set and the sequences of 

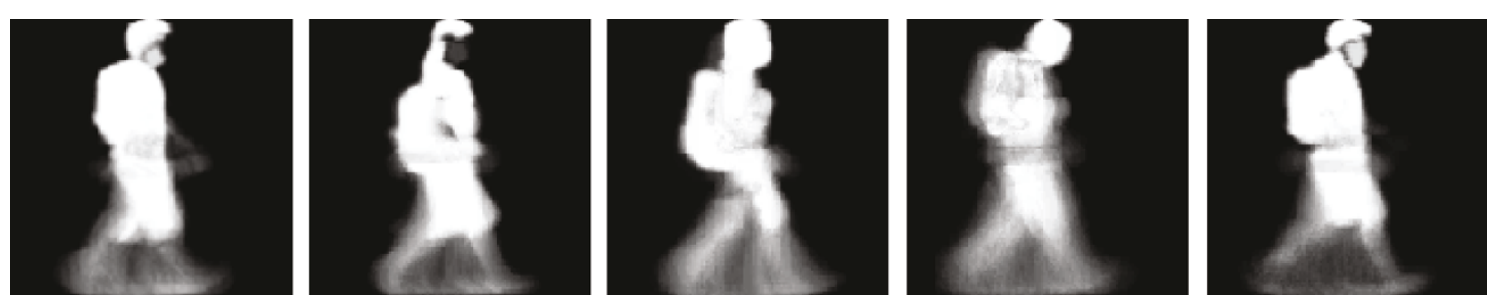

(a)
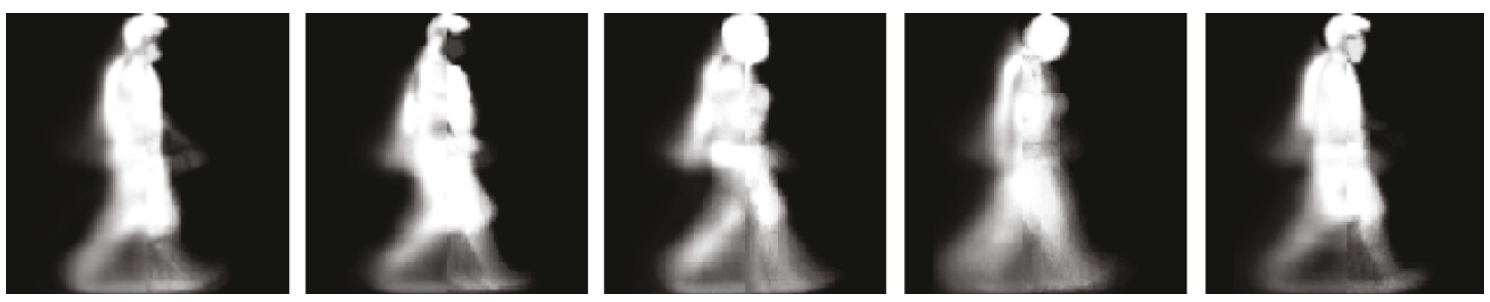

(b)

FIGURE 5: Result of backpack removal using recursive PCA reconstruction: (a) gait input images with backpack and (b) backpack removed images by recursive PCA reconstruction.

TABle 1: Training set designed for PCA and 1-NN.

\begin{tabular}{lcc}
\hline & $\begin{array}{c}\text { Training set of } \\
\text { PCA }\end{array}$ & $\begin{array}{c}\text { Training set of } \\
1-N N\end{array}$ \\
\hline Normal walking sequences & $153 \times 4=612$ & $153 \times 4=612$ \\
Slow walking sequences & $153 \times 2=306$ & $153 \times 0=0$ \\
Quick walking sequences & $153 \times 2=306$ & $153 \times 0=0$ \\
Total training sequences & 1224 & 612 \\
\hline
\end{tabular}

TABLE 2: Correct classification rate (CCR).

\begin{tabular}{lc}
\hline Backpack removal methods & CCR \\
\hline GEI [15] & 0.6000 \\
Simple PCA & 0.7059 \\
Simple PCA + combining process & 0.8105 \\
Recursive PCA & 0.8268 \\
\hline
\end{tabular}

walking with a backpack as the test set. The training sets are summarized in Table 1.

The performance of the proposed methods is reported in terms of the correct classification rate (CCR). In Table 2, "Simple PCA + combining process" denotes the method in which the left half of the PCA backpack removal is combined with the right half of the original GEI. As expected, the proposed backpack removal methods outperform the simple GEI in terms of CCR since they remove the backpack, which negatively affects the performance of the gait recognition system. Further, "Simple PCA + combining process" and "Recursive PCA" demonstrate better performance than the "Simple PCA" and increase the reliability of the gait recognition system by compensating for the backpack traces more smoothly, which are spread out over the entire reconstructed images.

Finally, we compare the performance of the proposed method with those of the previous methods: HTI [15],
TABLE 3: Comparison of several algorithms of the CASIA infrared night gait dataset.

\begin{tabular}{lccc}
\hline Methods & CCR & $\begin{array}{c}\text { Gallery } \\
\text { sequences }\end{array}$ & $\begin{array}{c}\text { Probe } \\
\text { sequences }\end{array}$ \\
\hline HTI [15] & 0.5100 & 184 & 92 \\
ODP [20] & Below 0.2000 & 306 & 306 \\
NDDP [21] & Below 0.2000 & 459 & 306 \\
UF [22] & Below 0.4000 & 306 & 306 \\
\hline Proposed method* & 0.7516 & 306 & 306 \\
Proposed method** & 0.7810 & 459 & 306 \\
Proposed method*** & 0.8268 & 612 & 306 \\
\hline
\end{tabular}

* Recursive PCA using two normal sequences as training data

** Recursive PCA using three normal sequences as training data

*** Recursive PCA using four normal sequences as training data

orthogonal diagonal projections [20], normalized dual diagonal projections [21], and uniprojective features [22]. The performances of the previous methods are cited directly from other research $[15,20-22]$ and compared with that of our method using the recursive PCA in Table 3. The CCR is only read out in the cumulative match score graph in [20-22], and the values are not precise. In the previous works, only the first two normal walking sequences of each individual were used as training data in $[20,22]$, and the first three were used in [21] as training data of 1-NN. For a fair comparison, we report three versions of results for our method depending on how many normal walking sequences were used as training data. In [15], a fraction of the data was selected randomly and used as training data, but this was not duplicated in our experiment. The experimental results are shown in Table 3.

In Table 3, we denote the orthogonal diagonal projections [20], normalized dual diagonal projections [21], and uniprojective features [22] as ODP, NDDP, and UF, respectively. It can be observed from Table 3 that our backpack 
removal method demonstrates a significant improvement in performance over the previous methods and makes gait a more reliable biometric.

\section{Conclusion}

Gait representations are obviously of importance in the gait recognition system, and a backpack is one of the most significant factors which deform the gait representation and negatively affect the performance of gait recognition systems. In this paper, backpack removal methods have been proposed for efficient gait recognition. We applied simple and recursive PCA reconstructions and the associated error compensation method to GEIs. Using the fact that the representational power of PCA depends on the training set, we successfully removed the backpack from gait representation images of people carrying a backpack. The proposed method was tested with CASIA C and demonstrated better performance than previous methods.

\section{Acknowledgment}

This work was supported by the Korea Science and Engineering Foundation (KOSEF) through the Biometrics Engineering Research Center (BERC) at Yonsei University. R112002105090020 (2008).

\section{References}

[1] N. V. Boulgouris and Z. X. Chi, "Gait recognition using radon transform and linear discriminant analysis," IEEE Transactions on Image Processing, vol. 16, no. 3, pp. 731-740, 2007.

[2] M. Murray, A. Drought, and R. Kory, "Walking patterns of normal men," Journal of Bone and Joint Surgery, vol. 46, no. 2, pp. 335-360, 1964.

[3] X. Li, S. J. Maybank, S. Yan, D. Tao, and D. Xu, "Gait components and their application to gender recognition," IEEE Transactions on Systems, Man and Cybernetics Part C, vol. 38, no. 2, pp. 145-155, 2008.

[4] X. Zhou and B. Bhanu, "Integrating face and gait for human recognition at a distance in video," IEEE Transactions on Systems, Man, and Cybernetics, Part B, vol. 37, no. 5, pp. 11191137, 2007.

[5] A. I. Bazin and M. S. Nixon, "Gait verification using probabilistic methods," in Proceedings of the 7th IEEE Workshop on Applications of Computer Vision (WACV '05), pp. 60-65, January 2007.

[6] A. F. Bobick and A. Y. Johnson, "Gait recognition using static, activity-specific parameters," in Proceedings of the IEEE Computer Society Conference on Computer Vision and Pattern Recognition (CVPR '01), vol. 1, pp. 423-430, 2001.

[7] C. BenAbdelkader, R. Culter, H. Nanda, and L. Davis, "EigenGait: motion-based recognition of people using image self-similarity," in Proceedings of the International Conference on Audio-and Video-Based Biometric Person Authentication (AVBPA '01), pp. 284-294, 2001.

[8] J. Han and B. Bhanu, "Individual recognition using gait energy image," IEEE Transactions on Pattern Analysis and Machine Intelligence, vol. 28, no. 2, pp. 316-322, 2006.

[9] T. H. W. Lam and R. S. T. Lee, "A new representation for human gait recognition: motion silhouettes image (MSI)," in Proceedings of the International Conference on Biometrics (ICB '06), vol. 3832 of Lecture Notes in Computer Science, pp. 612-618, 2006.

[10] A. F. Bobick and J. W. Davis, "The recognition of human movement using temporal templates," IEEE Transactions on Pattern Analysis and Machine Intelligence, vol. 23, no. 3, pp. 257-267, 2001.

[11] D. Xu, S. Yan, D. Tao, L. Zhang, X. Li, and H.-J. Zhang, "Human gait recognition with matrix representation," IEEE Transactions on Circuits and Systems for Video Technology, vol. 16, no. 7, pp. 896-903, 2006.

[12] D. Tao, X. Li, X. Wu, and S. J. Maybank, "General tensor discriminant analysis and gabor features for gait recognition," IEEE Transactions on Pattern Analysis and Machine Intelligence, vol. 29, no. 10, pp. 1700-1715, 2007.

[13] X. Li, S. Lin, S. Yan, and D. Xu, "Discriminant locally linear embedding with high-order tensor data," IEEE Transactions on Systems, Man, and Cybernetics, Part B, vol. 38, pp. 342-352, 2008.

[14] S. Hong, H. Lee, I. F. Nizami, and E. Kim, "A new gait representation for human identification: mass vector," in Proceedings of the 2nd IEEE Conference on Industrial Electronics and Applications (ICIEA '07), pp. 669-673, May 2007.

[15] D. Tan, K. Huang, S. Yu, and T. Tan, "Efficient night gait recognition based on template matching," in Proceedings of the 18th International Conference on Pattern Recognition, vol. 3, pp. 1000-1003, 2006.

[16] Y. Saito, Y. Kenmochi, and K. Kotani, "Estimation of eyeglassless facial images using principal component analysis," in Proceedings of the IEEE International Conference on Image Processing (ICIP '99), vol. 4, pp. 197-201, Kobe, Japan, October 1999.

[17] J.-S. Park, Y. H. Oh, S. C. Ahn, and S.-W. Lee, "Glasses removal from facial image using recursive error compensation," IEEE Transactions on Pattern Analysis and Machine Intelligence, vol. 27, no. 5, pp. 805-811, 2005.

[18] K. Bashir, T. Xiang, and S. Gong, "Feature selection on gait energy image for human identification," in Proceedings of the IEEE International Conference on Acoustics, Speech and Signal Processing (ICASSP '08), pp. 985-988, March-April 2008.

[19] D. Tao, X. Li, X. Wu, and S. J. Maybank, "Human carrying status in visual surveillance," in Proceedings of the IEEE Computer Society Conference on Computer Vision and Pattern Recognition (CVPR '06), vol. 2, pp. 1670-1677, 2006.

[20] D. Tan, K. Huang, S. Yu, and T. Tan, "Orthogonal diagonal projections for gait recognition," in Proceedings of the International Conference on Image Processing (ICIP '07), vol. 1, pp. 337-340, September-October 2007.

[21] D. Tan, S. Yu, K. Huang, and T. Tan, "Walker recognition without gait cycle estimation," in Proceedings of the International Conference on Biometrics (ICB '07), Lecture Notes in Computer Science, pp. 222-231, 2007.

[22] D. Tan, K. Huang, S. Yu, and T. Tan, "Uniprojective features for gait recognition," in Proceedings of the International Conference on Biometrics (ICB '07), Lecture Notes in Computer Science, pp. 673-682, 2007. 